\title{
Opposite Effects of Vitamin C and Vitamin E on the Antifungal Activity of Honokiols
}

\author{
Lingmei Sun ${ }^{1 *}$, Xiaolong Ye ${ }^{2}$, Dafa Ding ${ }^{2}$, and Liao Kai ${ }^{3 *}$ \\ ${ }^{1}$ Department of Pharmacology, Medical School of Southeast University, Nanjing, 210009, P.R. China \\ ${ }^{2}$ Department of Endocrinology, The Second Affiliated Hospital of Nanjing Medical University, Nanjing Medical University, Nanjing, \\ 210000, P.R. China \\ ${ }^{3}$ Department of Pathology and Pathophysiology, Medical School of Southeast University, Nanjing, 210009, P.R. China
}

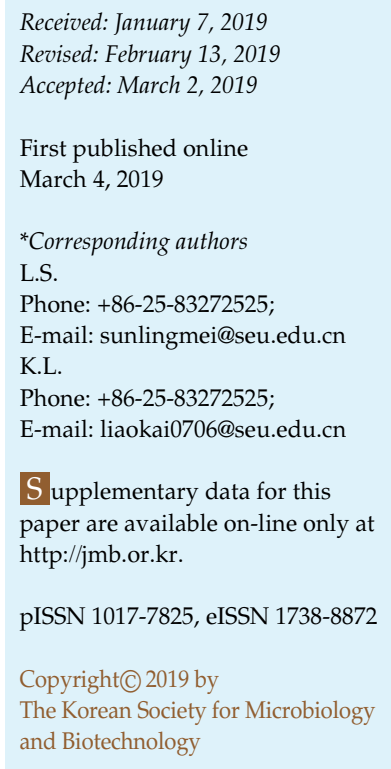

The aim of the present study was to evaluate the effects of two well-known natural antioxidants, vitamin C (VC) and vitamin E (VE), on the antifungal activity of honokiol against Candida albicans. The broth microdilution method was employed to test the antifungal activities of honokiol with or without antioxidants in the medium against C. albicans strain. Intracellular reactive oxygen species and lipid peroxidation were determined by fluorescence staining assay. Mitochondrial dysfunction was assessed by detecting the mitochondrial DNA and the mitochondrial membrane potential. We observed that VC could significantly potentiate the antifungal activities of honokiol while VE reduced the effectiveness of honokiol against $C$. albicans. In addition, VC accelerated honokiol-induced mitochondrial dysfunction and inhibited glycolysis leading to a decrease in cellular ATP. However, VE could protect against mitochondrial membrane lipid peroxidation and rescue mitochondrial function after honokiol treatment. Our research provides new insight into the understanding of the action mechanism of honokiol and VC combination against C. albicans.

Keywords: Candida albicans, honokiol, antioxidant, oxidative stress, glycolysis

\section{Introduction}

Reactive oxygen species (ROS) are not only produced by normal cellular respiration processes, but also by compounds used as therapeutic drugs, which interfere with oxygen metabolism [1]. The over-accumulation of ROS can initiate the deleterious effects on cellular biomolecules such as protein, lipid, RNA, and DNA, and result in cell death [1]. Cells normally have the ability to protect themselves against ROS-mediated damage by intracellular antioxidant enzymes to maintain the homeostasis of ROS at a low concentration [2]. Antioxidants such as vitamin C (ascorbic acid, VC) and $E(\alpha$-tocopherol, VE) can terminate free radicals by eliminating the unpaired condition of the radical $[2,3]$. However, some researchers have put forward the paradox that VC and VE may have both pro- and antioxidant activities [4]. VC is soluble in water (hydrophilic) and VE in the lipids (lipophilic). They will directly react with or neutralize ROS such as hydrogen peroxide [4]. After that, VE itself becomes $\alpha$-tocopheroxyl radicals while VC changes into a new radical (Asc ${ }^{-}$). Under these conditions, antioxidants exhibit pro-oxidant effects and can become harmful $[2,4]$. VC itself is readily regenerated from Asc $^{-}$with the help of NADH or NADPH-dependent reductases. In addition, the cytotoxicity of ascorbate in the presence of metal ions has been interpreted, from Fenton's reaction, to occur via the generation of oxygen-derived free radicals $[4,5]$. Previous studies have shown that VC inhibits glyceraldehyde-3-phosphate dehydrogenase (GAPDH) and decreases the cellular glutathione level, leading to high ROS generation and oxidative stress [6].

Honokiol, a polyphenol compound present in the traditional Chinese medicine Magnolia officinalis Rehd. et Wils. bark, is an interesting small-molecule having various 
pharmacological activities, including anti-tumor, antioxidation, anti-inflammatory, and anti-microbial activity as well as protection against neurotoxicity, hepatotoxicity, and thrombosis in preclinical experimental models [7-9]. Honokiol contains two phenolic groups that can exhibit antioxidant properties [7]. Previous mechanism studies, including those from our own laboratory, indicated that honokiol-induced apoptosis in C. albicans was associated with ROS production, which indicated the pro-oxidant activities of honokiol [10, 11]. In the present study, we investigated the effects of two well-known natural antioxidants, $\mathrm{VC}$ and $\mathrm{VE}$, on the antifungal activity of honokiol against $C$. albicans.

\section{Materials and Methods}

\section{Materials}

Honokiol (5,5'-diallyl-2,4'-dihydroxybiphenyl) was purchased from Xi'an Yuquan Biological Technology Co., Ltd China and its purity is over $98 \%$. VC and VE were obtained from Aladdin BioChem Technology Co., LTD (China) and their purities are over 99\% and $96 \%$, respectively. VC and VE were dissolved in water and DMSO, respectively. DCFH-DA ( $2^{\prime}, 7^{\prime}$-dichlorofluorescein diacetate), DPPP (diphenyl-1-pyrenylphosphine), DAPI, JC-1 (5,50,6,60-tetrachloro1,10,3,30-tetraethylbenzimidazolocarbocyanine iodide), and other molecular grade chemicals were obtained from Sigma Chemicals (USA).

\section{Microorganisms}

The C. albicans strains SC5314, YEM30, and Tom70-GFP strain were cultured in YPD (yeast extract/peptone/dextrose) broth [10, 12]. For agar plates, $2 \%(\mathrm{w} / \mathrm{v})$ bacto agar (Difco, BD Biosciences, USA) was added to the medium. The strains were stored as frozen stock with $15 \%(\mathrm{v} / \mathrm{v})$ glycerol at $-80^{\circ} \mathrm{C}$. Before each experiment, cells were freshly revived on YPD plate from the stock.

\section{Sensitivity Determination}

The sensitivities of $C$. albicans to honokiol and antioxidants were tested by the broth microdilution method according to the CLSI standard M27-A3 with final inoculum of $0.5 \times 10^{3}$ to $2.5 \times 10^{3}$ cells $/ \mathrm{ml}$ [13]. Quality control was performed with the antifungal agent fluconazole on each day of testing using the CLSI-recommended reference strain C. albicans ATCC 10231. The assay was performed with RPMI 1640 medium in 96-well microtitration plates (Costar,Corning, NY, USA). The minimum inhibitory concentrations (MICs) were determined by optical density measured at $600 \mathrm{~nm}$ with a BioTek Synergy 4 multidetection microplate reader (Synergy HT; BioTek, USA) after incubation at $35^{\circ} \mathrm{C}$ for $48 \mathrm{~h}$. The MICs were determined as the concentrations of drug that inhibited growth by $80 \%$ compared to that of cells grown in the control.

\section{Time-Kill Test}

Time-kill tests were performed as described previously [14]. C. albicans SC5314 cells with starting inoculum of $10^{5}$ cells $/ \mathrm{ml}$ were exposed by different concentrations of antioxidants or honokiol. Samples were taken at 3, 6, 9, 12, and $24 \mathrm{~h}$ and the viabilities were determined by spot assay. Using a $20-\mu \mathrm{l}$ sampling volume, the limit of detection was $50 \mathrm{CFU} / \mathrm{ml}$. Synergy was defined as $\mathrm{a} \geq 2 \log _{10} \mathrm{CFU} / \mathrm{ml}$ decrease after $24 \mathrm{~h}$ with the combination compared with honokiol alone; indifference was defined as a $<2 \log _{10} \mathrm{CFU} / \mathrm{ml}$ increase or decrease at $24 \mathrm{~h}$ with the combination compared with that of honokiol alone; and antagonism was a $\geq 2 \log _{10} \mathrm{CFU} / \mathrm{ml}$ increase after $24 \mathrm{~h}$ with the combination compared with that of honokiol alone [14]. Fungistatic effect was defined as a decrease $<3 \log _{10} \mathrm{CFU} / \mathrm{ml}$ compared to the initial inoculum and fungicidal as a decrease $\geq 3 \log _{10} \mathrm{CFU} / \mathrm{ml}$ [14].

\section{ROS Determination}

SC5314 cells were diluted to $1 \times 10^{7}$ cells $/ \mathrm{ml}$ using YPD medium and exposed to VC $(10 \mathrm{mM}), \mathrm{VE}(10 \mathrm{mM})$, honokiol $(60 \mu \mathrm{M})$, the combinations, or the DMSO vehicle control at $35^{\circ} \mathrm{C}$ for $4 \mathrm{~h}$. Intracellular ROS concentrations were determined using DCFH-DA staining method as previously described [10].

\section{Cell Staining with DPPP}

C. albicans SC5314 cells were diluted to $1 \times 10^{7}$ cells $/ \mathrm{ml}$ with YPD medium and exposed to VC $(10 \mathrm{mM}), \mathrm{VE}(10 \mathrm{mM})$, honokiol $(60 \mu \mathrm{M})$, the combinations, or the DMSO vehicle control at $35^{\circ} \mathrm{C}$ for $4 \mathrm{~h}$. The cell suspensions were stained with $10 \mu \mathrm{M}$ DPPP at $37^{\circ} \mathrm{C}$ in the dark for $10 \mathrm{~min}$ [15]. The images were taken by a fluorescence microscope (Olympus IX71, Olympus Co., Japan) with a $340 / 380 \mathrm{~nm}$ excitation filter.

\section{DAPI Staining}

C. albicans SC5314 cells were adjusted to $1 \times 10^{7}$ cells $/ \mathrm{ml}$ in YPD medium and exposed to VC $(10 \mathrm{mM}), \mathrm{VE}(10 \mathrm{mM})$, honokiol $(60 \mu \mathrm{M})$, the combinations, or the DMSO vehicle control at $35^{\circ} \mathrm{C}$ for $4 \mathrm{~h}$. After that, cells were fixed with $70 \%$ ethanol for $10 \mathrm{~min}$, and then stained with $5 \mu \mathrm{g} / \mathrm{ml}$ DAPI in the dark for $10 \mathrm{~min}$. The images were taken by the fluorescence microscope with a 340 / $380 \mathrm{~nm}$ excitation filter.

\section{JC-1 Assay}

The membrane-permeant JC- 1 dye is widely used to detect mitochondrial membrane potential $(\mathrm{mt} \Delta \psi)$. Polarized mitochondria are marked by punctate orange-red fluorescent staining [16]. The orange-red punctate staining is replaced by diffuse green monomer fluorescence during mitochondria depolarization [16]. SC5314 cells were diluted to $1 \times 10^{7}$ cells $/ \mathrm{ml}$ with YPD medium and exposed to VC $(10 \mathrm{mM}), \mathrm{VE}(10 \mathrm{mM})$, honokiol $(60 \mu \mathrm{M})$, the combinations, or the DMSO vehicle control at $35^{\circ} \mathrm{C}$ for $4 \mathrm{~h}$. C. albicans cells were stained by $5 \mu \mathrm{M} \mathrm{JC}-1$ at $37^{\circ} \mathrm{C}$ for $30 \mathrm{~min}$ [17]. 
JC-1 green and red fluorescence was detected by flow cytometry (Becton Dickinson, Franklin Lakes, NJ, USA) recorded on FL1 and FL2 channels, respectively. The dots were distributed in the upper right quadrant (UR) and lower right quadrant (LR). High $\mathrm{mt} \Delta \psi$ is identified as the \% of cells emitting fluorescence signals in the UR quadrant and low $\mathrm{mt} \Delta \psi$ is identified as the \% of cells emitting fluorescence signals in the LR quadrant.

\section{Measurement of Intracellular ATP Levels}

$C$ albicans strain SC5314 was diluted to $1 \times 10^{7}$ cells $/ \mathrm{ml}$ in YPD broth. After cell treatment with VC $(10 \mathrm{mM})$ or VE $(10 \mathrm{mM})$, honokiol $(60 \mu \mathrm{M})$, the combinations, or the DMSO vehicle control for $4 \mathrm{~h}$, the cells were washed twice with cold PBS. And then, the cells were suspended with ATP-releasing reagent provided by the ATP assay kit (Beyotime Institute of Biotechnology, China), mixed with glass beads (0.45-0.5 mm diameter), and broken by a FastPrep homogenizer (FastPrep FP120; Savant Instruments, USA). The homogenates were centrifuged at $10000 \mathrm{~g}$ for $45 \mathrm{~min}$ to separate cell-free extracts. Intracellular ATP levels were determined by using a luciferase-based assay, according to the instructions of the manufacturer [18].

\section{Measurement of Tdh3 Expression}

C. albicans expressing GFP-TDH3 cells diluted to $1 \times 10^{7}$ cells $/ \mathrm{ml}$ in YPD broth were treated with VC $(10 \mathrm{mM})$ or VE $(10 \mathrm{mM})$, honokiol $(60 \mu \mathrm{M})$, the combinations, or the DMSO vehicle control for $4 \mathrm{~h}$ [19]. The expression of Tdh3 was indicated by the fluorescence intensity of GFP measured by fluorescence microscope and flow cytometry [19].

\section{Statistical Analysis}

The data represent the average of three separate experiments and each was performed in triplicate. All data were presented as means \pm standard error of mean (SEM). Graphs were generated using Microsoft Excel (Microsoft Corp., Redmond, WA, USA). Statistical analysis was performed using SPSS 12.0 (SPSS Inc., USA). Differences between groups were determined using analysis of variance (ANOVA). A $p$ value of $<0.05$ was considered statistically significant.

\section{Results}

Effects of VC and VE on the Antifungal Activity of Honokiol against $C$. albicans

The MICs for honokiol, VC, and VE against C. albicans SC5314 were $120 \mu \mathrm{M}$, > $50 \mathrm{mM}$, and > $50 \mathrm{mM}$, respectively. Previous studies indicated that honokiol could induce apoptosis in C. albicans, associated with production of ROS [10]. To test if antioxidants like VC and VE could protect C. albicans from oxidative damage after honokiol exposure, SC5314 cells were incubated with VC $(10 \mathrm{mM})$ and VE $(10 \mathrm{mM})$ alone or in combination with honokiol $(30,60$, and
$120 \mu \mathrm{M})$. VC did not reduce, in fact enhanced, the honokiol-induced cell death in C. albicans cells (Fig. 1A). Given the initial inoculum of $10^{5}$ cells $/ \mathrm{ml}$, honokiol as a fungicide compound, exhibited no growth at MIC $(120 \mu \mathrm{M})$. While honokiol alone had no antifungal effect at $30 \mu \mathrm{M}$, when administered in combination with $10 \mathrm{mM}$ $\mathrm{VC}$, it induced a $1.35 \log _{10} \mathrm{CFU} / \mathrm{ml}$ decrease, compared with honokiol administrated alone at $24 \mathrm{~h}$. Honokiol $(60 \mu \mathrm{M})-\mathrm{VC}(10 \mathrm{mM})$ combination exhibited even better fungicidal activity ( $>3 \log _{10} \mathrm{CFU} / \mathrm{ml}$ decrease compared with starting inoculum) by $24 \mathrm{~h}$ (Fig. 1A). In addition, a synergistic interaction between honokiol $(60 \mu \mathrm{M})$ and VC $(10 \mathrm{mM})$ was also confirmed according to the definition of synergistic interaction assessed by time-kill assay. After $24 \mathrm{~h}$, the growth of the honokiol-vitamin C combination group was accelerated but the $\log _{10} \mathrm{CFU} / \mathrm{ml}$ were still lower than when the individual compounds were used alone (data not shown). In contrast, VE could effectively protect C. albicans from honokiol-induced cell death, even when combined with the fungicidal concentration of $120 \mu \mathrm{M}$ of honokiol (Fig. 1B). Hence the antagonism was observed when honokiol $(120 \mu \mathrm{M})$ was combined with VE $(10 \mathrm{mM})$. The same phenomenon has been observed in another C. albicans strain YEM30 (Fig. S1). We next investigated whether a 1-h pretreatment with VC and VE could protect $C$. albicans from honokiol-induced cell death. Pretreatment with VC $(10 \mathrm{mM})$ could not attenuate the antifungal activity of honokiol while that with VE (10 mM) still hindered the efficacy of honokiol treatment (Fig. 1C). In order to further verify the opposite effects of VC and VE on the antifungal activity of honokiol against C. albicans, different concentrations (lower than MICs) of both vitamins, ranging from 0.3125 to $40 \mathrm{mM}$, were tested. Even at the lowest concentration of $\mathrm{VC}(0.3125 \mathrm{mM})$, the antifungal activities of honokiol (at 30 or $60 \mu \mathrm{M}$ ) were significantly enhanced, compared to the situation when the individual compounds were used alone (Fig. 1D). The fungicidal activity of honokiol $(60 \mu \mathrm{M})$ was noted with $\mathrm{VC}(\geq 10 \mathrm{mM})$ in a concentration-dependent manner. In addition, there is a dose-dependent protective effect on C. albicans when honokiol is used in combination with VE (Fig. 1E).

\section{Effects of VC and VE on ROS Generation and Mitochondrial Lipid Peroxidation after Honokiol Exposure}

VC and VE are perhaps the most well-known antioxidants because of their ability to scavenge ROS [4]. The antifungal mechanism of honokiol was associated with mitochondrial dysfunction accompanied with ROS production [10]. We examined the effects of antioxidants, VC and VE (both at 
A
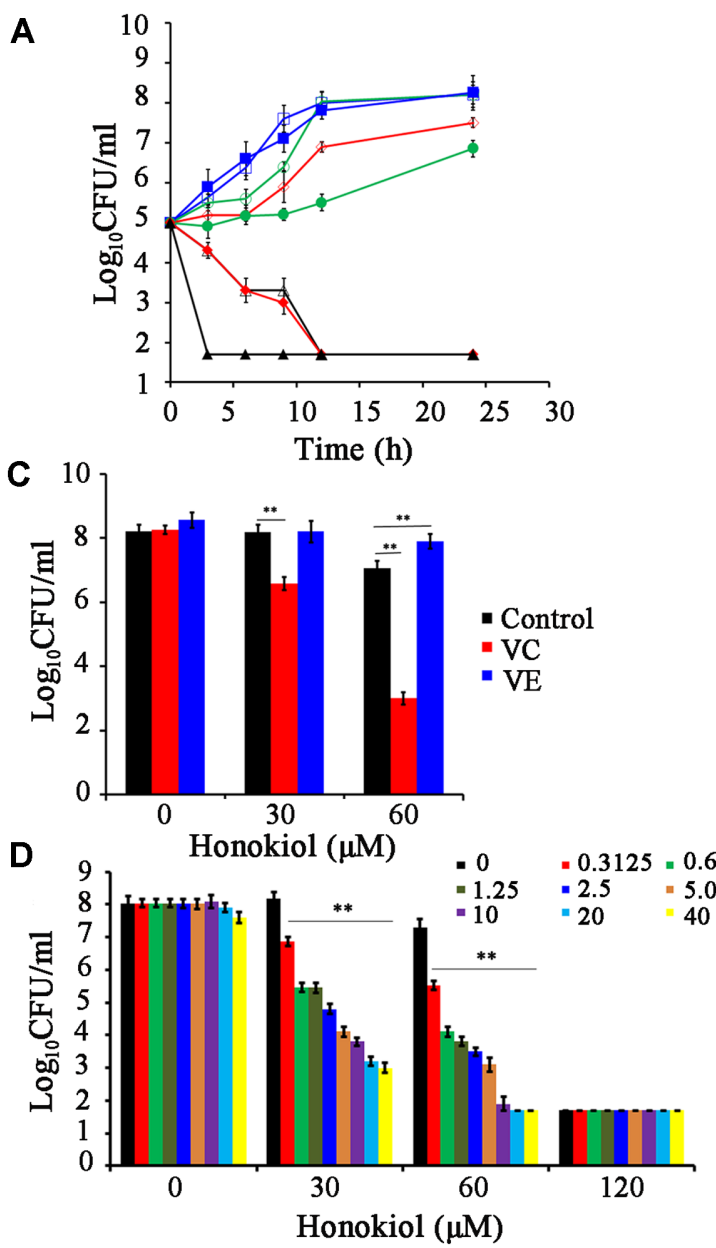

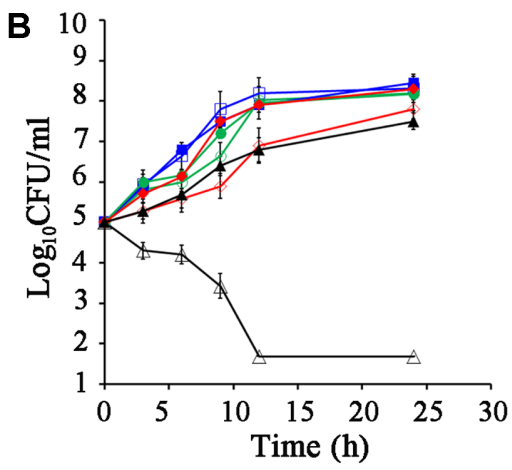

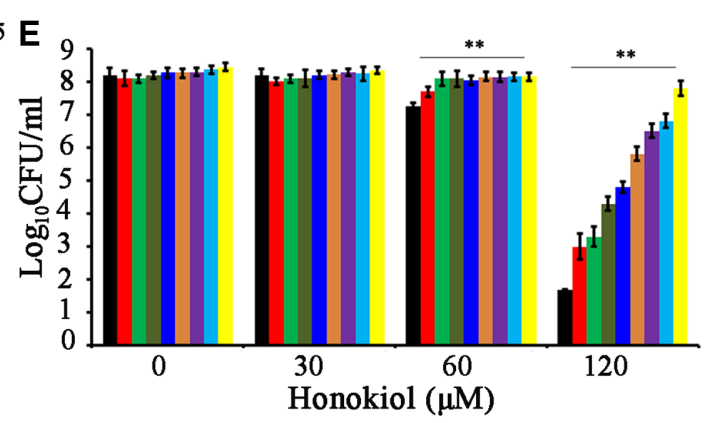

Fig. 1. Effects of VC and VE on the antifungal activities of honokiol.

(A and B) Growth curve of C. albicans SC5314 cells with either $10 \mathrm{mM} \mathrm{VC} \mathrm{(A)} \mathrm{or} 10 \mathrm{mM}$ VE (B) on the antifungal activities of honokiol. ( $\square$ ) control, $(\bigcirc) 30 \mu \mathrm{M}$ honokiol, $(\diamond) 60 \mu \mathrm{M}$ honokiol, $(\triangle) 120 \mu \mathrm{M}$ honokiol, $(\boldsymbol{\square}) \mathrm{VC}$ or VE, $(\bullet) 30 \mu \mathrm{M}$ honokiol with VC or VE, $(\diamond) 60 \mu \mathrm{M}$ honokiol with VC or VE, ( $\Delta) 120 \mu \mathrm{M}$ honokiol with VC or VE. (C) Effects of pretreatment of C. albicans SC5314 cells with either VC or VE on the antifungal activities of honokiol. The SC5314 cells were treated with VC or VE both at concentrations $\mathrm{mM}$ of $10 \mathrm{mM}$ for $1 \mathrm{~h}$ and then exposed to honokiol for $24 \mathrm{~h}$. The cell viability was determined by spot assay. (D and E) Effects of varying concentrations of VC (D) and VE (E) on the antifungal activities of honokiol against C. albicans SC5314 cells. ${ }^{* *}, p<0.01$ vs. honokiol exposure alone.

$10 \mathrm{mM}$ ), on honokiol-induced ROS generation. The levels of ROS were detected by DCFH-DA staining. Fig. 2A shows the VC- and VE-administered group had no green fluorescence in $C$. albicans loaded with DCFH-DA. Honokiol $(60 \mu \mathrm{M})$ induced DCF-positive staining in 36.0\% $\pm 10.0 \%$ cells while VC supplement resulted in a 1.8 -fold increase of ROS positive cells compared to that in honokiol alone (Fig. 2B). When supplemented with VE, honokiolinduced ROS generation was decreased 6.5-fold, indicating that VE could effectively defend the cells against honokiolinduced ROS generation (Fig. 2B). Mitochondrion is the primary source of ROS, such as the superoxide anion radicals, so it might also be a primary target for the damaging effects of ROS, like mitochondrial membrane lipid peroxidation. DPPP is an agent that reacts with lipid peroxidations to generate the fluorescent product DPPP oxide [20]. To elucidate the lipid peroxidation on the mitochondrial membrane, C. albicans expressing Tom70 (a constitutive protein of mitochondria)-GFP were analyzed in this study. Tom70-GFP strain cells were stained with DPPP after honokiol exposure. We observed that there was a co-localization of the fluorescent product, DPPP oxide, on the mitochondria, hence suggesting honokiol-induced ROS generation and stimulation of lipid peroxidation in mitochondria (Fig. S2). Compared to the honokiol exposure alone, VC-supplemented honokiol induced a 1.5-fold 


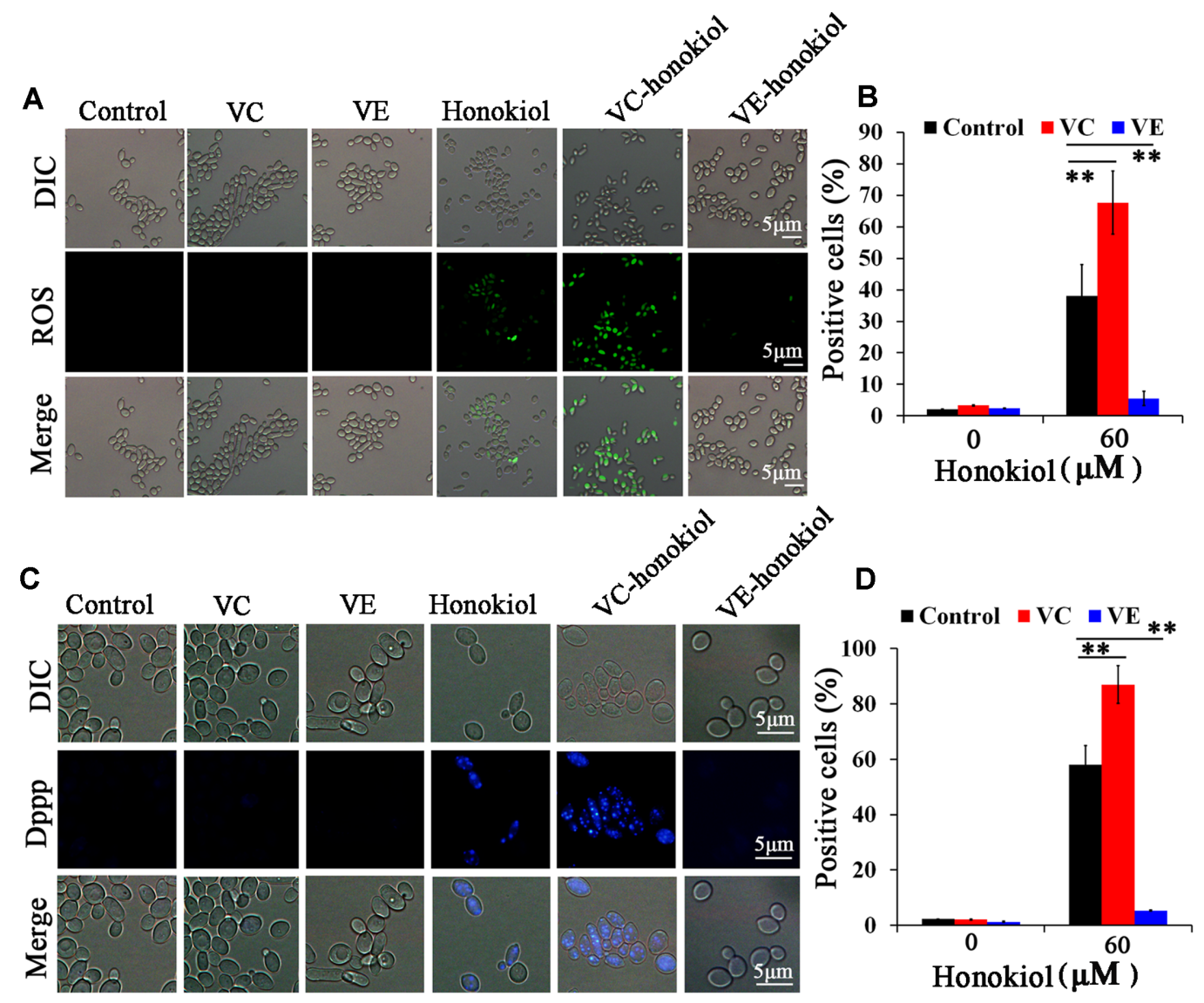

Fig. 2. Effects of VC and VE on the ROS production and oxidation of lipids after honokiol exposure.

(A) ROS production was observed by fluorescence microscope. (B) The percentage of ROS-positive cells after honokiol exposure. Fluorescent images (C) and the percentage of DPPP-positive cells (D). VC and VE, $10 \mathrm{mM}$; honokiol, $60 \mu \mathrm{M}$. ${ }^{* *}, p<0.01$ vs. honokiol exposure alone.

increase in the percentage of DPPP positive cells (Figs. 2C and 2D). However, when supplemented with VE, honokiolinduced lipid peroxidation was almost completely reversed (Figs. 2C and 2D).

\section{Effects of VC and VE on mtDNA and $m t \Delta \psi$ after Honokiol Exposure}

Because of the lack of protective histones and the close proximity to the electron transport chain, the major locus for free radical production, mtDNA is especially sensitive to ROS attack [1]. DAPI stain is effective for visualization of nuclear DNA and mtDNA in a variety of organisms [21]. Staining with DAPI revealed small fluorescent spots in the cytoplasm for mtDNA aggregates in the control group, when observed under fluorescence microscope (Fig. 3A). $\mathrm{VC}$ or VE administered alone had almost no effect on mtDNA in C. albicans. VC-supplemented honokiol, however, showed extremely condensed nuclei with cytoplasm completely devoid of mtDNA while VE-supplement could prevent the honokiol-induced damage to mtDNA (Fig. 3A).

In addition, we used JC-1 dye to monitor mitochondrial health. The depolarization of mitochondria is indicated by a decrease in the red/green fluorescence intensity ratio [16]. As expected, consistent with a mitochondrial localization, the red fluorescence was mostly found throughout the cytoplasm in the control, VC- and VEtreated group (Fig. 3B). Treatment with honokiol showed some diffused red fluorescence and increased green fluorescence. Honokiol supplemented with VC showed a noticeable increase in the green fluorescence and changed the distribution of red fluorescence which was dispersed in the cytoplasm, suggesting that the mitochondrial membrane was depolarized. However, VE supplement repressed the honokiol-induced changes of $\mathrm{mt} \Delta \psi$, as implicated by a reduction of green fluorescence and restoration of red fluorescence (Fig. 3B). The effect of VC and VE on $m t \Delta \psi$ in C. albicans after honokiol exposure was further confirmed by flow cytometry. As shown in Figs. 3C and 3D, honokiol, 

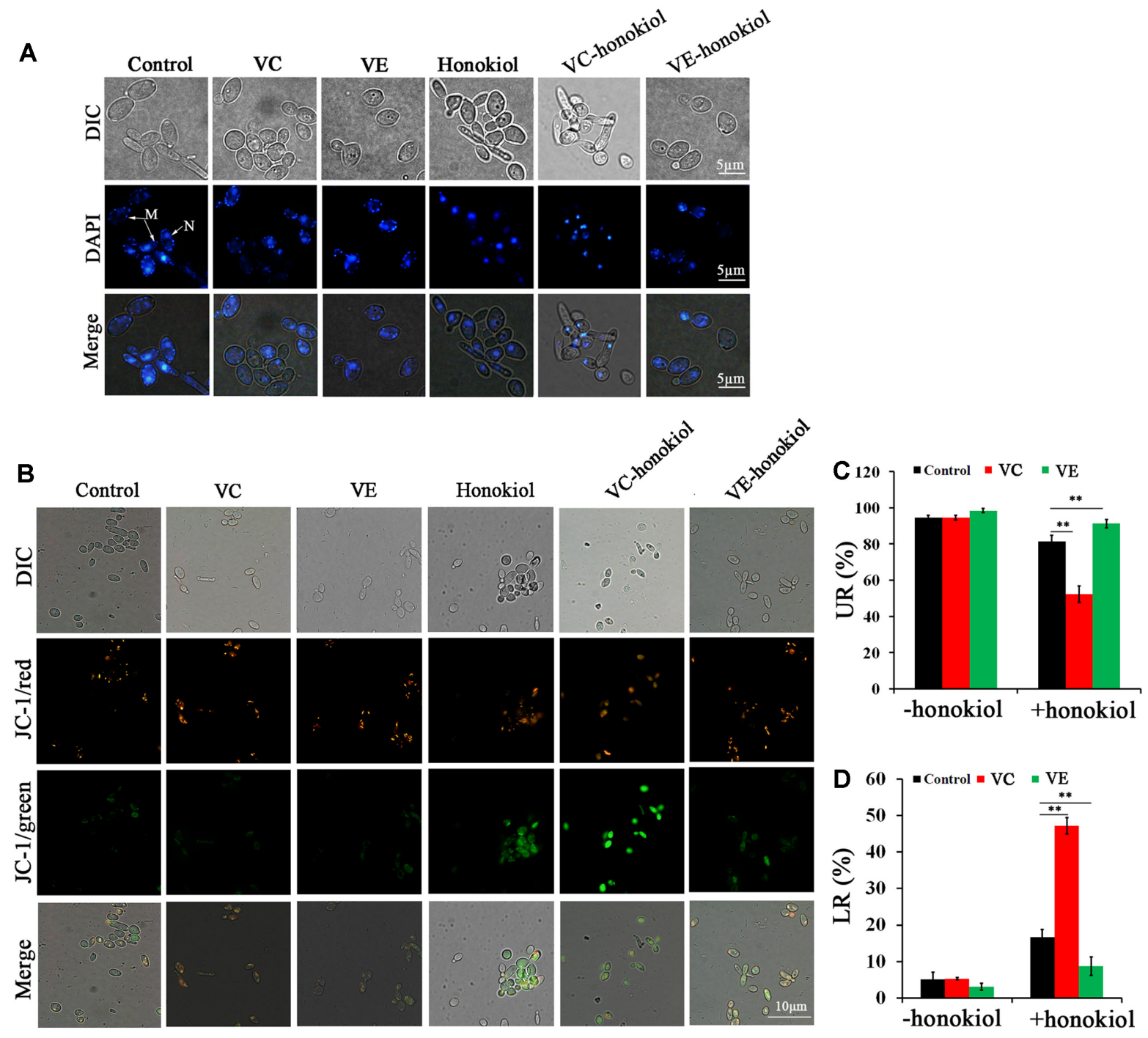

Fig. 3. Effects of VC and VE on mtDNA and $\mathrm{mt} \Delta \psi$ in C. albicans after honokiol exposure.

(A) Fluorescent images of C. albicans SC5314 cells stained with DAPI. N, nucleus; M, mtDNA. (B) Fluorescent images of C. albicans SC5314 cells stained with JC-1. (C and D) The $\mathrm{mt} \Delta \psi$ was measured by flow cytometry-High $\mathrm{mt} \Delta \psi$ is identified as the $\%$ of cells emitting fluorescence signals in the UR quadrant and low $\mathrm{mt} \Delta \psi$ is identified as the $\%$ of cells emitting fluorescence signals in the LR quadrant. ${ }^{* *}, p<0.01$ vs. honokiol exposure alone.

supplemented with VC, showed a significant fall in $\mathrm{mt} \Delta \psi$ compared to that when administered alone, whereas honokiol supplemented with VE attenuated the honokiolinduced collapse of $\mathrm{mt} \Delta \psi$ in C. albicans.

\section{Effects of VC and VE on Tdh3 Expression and ATP Level} in C. albicans after Honokiol Exposure

Glycolysis is important for maintaining the tricarboxylic acid (TCA) cycle, oxidative phosphorylation, and the mitochondrial function [22]. In C. albicans, TDH3 encodes an NAD-linked glyceraldehyde-3-phosphate dehydrogenase
(GAPDH), an enzyme of the glycolysis pathway. VC could induce oxidative stress and inhibits the activity of GAPDH [22]. We found that the expression of Tdh3 significantly increased after honokiol exposure (Figs. 4A and 4B). When supplemented with VC, the expression of Tdh3 was 1.6fold lower in honokiol-treated cells compared to vehicletreated ones (Fig. 4B). However, there was almost no change in the expression of Tdh3 between vitamin Ehonokiol-treated cells and vehicle-treated cells. ATP is one of the end products of glycolysis and TCA cycle/ photophosphorylation. There was no effect on the ATP 


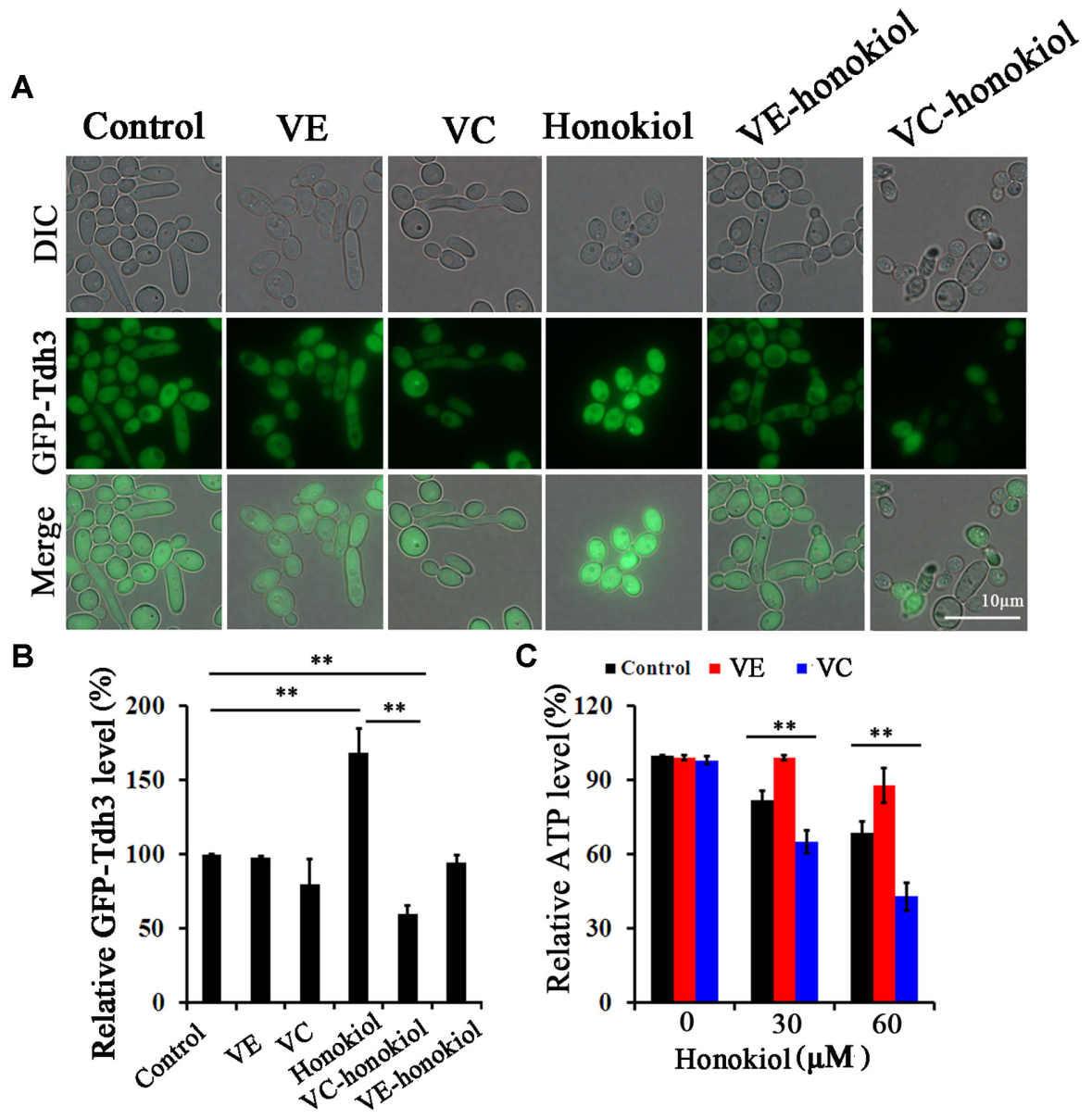

Fig. 4. Effects of VC and VE on Tdh3 expression and ATP level in C. albicans after honokiol exposure.

(A) Fluorescent images of C. albicans TDH3-GFP-CAI4 cells. (B) Relative GFP-Tdh3 level in C. albicans. (C) ATP level in C. albicans SC5314. $* *, p<0.01$.

production in VE- and VC-alone-treated cells when both used at a concentration of $10 \mathrm{mM}$ (Fig. 4C). Honokiol treatment, however, caused a significant decrease in ATP level and the drop of ATP level was increased when supplemented with VC (Fig. 4C). On the contrary, VE abolished the ability of honokiol to decrease the ATP level (Fig. 4C).

\section{Effect of Glycolysis Inhibitor 2-Deoxy-Glucose (2-DG) on the Antifungal Activity of Honokiol}

2-DG, a glucose analog, has been known to be used as a classical glycolysis inhibitor by targeting hexokinase [23]. 2-DG exhibits a cytotoxic effect in cancer cells, especially those with mitochondrial respiratory defects, or cells in a hypoxic environment [23]. Given the starting inoculum of $10^{5}$ cells $/ \mathrm{ml}$ and then incubation at $35^{\circ} \mathrm{C}$ for $24 \mathrm{~h}, 2-\mathrm{DG}$ $(24 \mu \mathrm{M})$ alone had almost no toxic effect against $C$. albicans
SC5314, but it significantly enhanced the antifungal activity of honokiol (60 $\mu \mathrm{M})$ (Fig. 5).

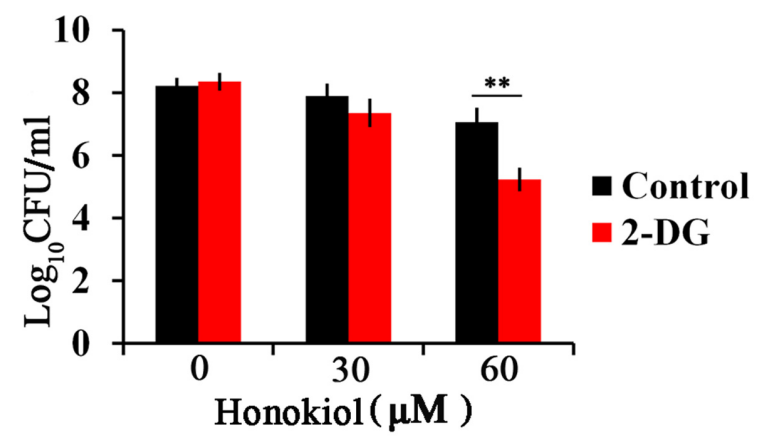

Fig. 5. Comparisons of the effects of 2-DG, honokiol, and the combination of 2-DG and honokiol on the viability of C. albicans. $* *, p<0.01$. 


\section{Discussion}

C. albicans, an opportunistic pathogen, can lead to infections ranging from mild mucosal lesions to lifethreatening systemic diseases depending on the physiological or immunological status of the host [24]. Because of the limited numbers of antifungal drugs, undesirable toxic side effects, and the emergence of resistant strains, the treatment of candidiasis faces an enormous clinical challenge [25]. However, some natural molecules showing promising antifungal properties could be considered as an important source for development of novel anti-Candida therapy [26-28]. During the last decade, honokiol has been reported to have potent anticancer and antimicrobial activities, including anti-Candida activities [12]. In our previous research, the action of honokiol against C.albicans was shown to be associated with mitochondrial dysfunction accompanied with ROS production [10]. A supplement with the inhibitor of superoxide dismutases increased efficacy of honokiol against C. albicans [10]. VC and VE, two well-known antioxidant nutrients, can protect cells from oxidative stress [4]. Therefore, some published studies support the idea that antioxidants VC or VE hinder the efficacy of chemotherapy treatment by disturbing the oxidative mechanisms of action [29]. However, it is very interesting that researchers, now, believe that antioxidant supplementation can actually potentiate the therapeutic index of amphotericin B [30].

In this study, we investigated the effect of VC and VE on the antifungal activity of honokiol against $C$. albicans. Interestingly, we observed the two vitamins to have opposite effects on the antifungal activity of honokiol. Our results showed VC supplement effectively enhanced the efficacy of honokiol against $C$. albicans, whereas in combination with $\mathrm{VE}$, we observed a protective effect against honokiol-induced oxidative stress in $C$. albicans (Fig. 1). The effects of pretreatment with $\mathrm{VC}$ on honokiol activity against $C$. albicans are the same as that of concomitant administration (Fig. 1). In this study, a wide range of concentrations of VC and VE were tested. We observed that even a lower concentration $(0.3125 \mathrm{mM})$ of VC was able to potentiate the antifungal activity of honokiol after $24 \mathrm{~h}$-incubation (Fig. 1). It has been reported that plasma VC concentrations, higher than $10 \mathrm{mM}$, could be easily achieved in humans and in the murine pharmacokinetic study without significant toxicity [22]. Our results provide a possible approach to explore the therapeutic use of VC-honokiol combination against Candida infection.

ROS being generated continuously by the mitochondrial respiratory chain causes the mitochondria to be particularly susceptible to ROS-induced damage [1]. Supplement with VC exacerbated the honokiol-induced disruption of mitochondrial function, as evaluated by ROS generation (Fig. 2), mitochondrial lipid peroxidation (Figs. S2 and 2), loss of mtDNA (Fig. 3), and $\mathrm{mt} \Delta \psi$ depolarization (Fig. 3). In contrast, supplement with VE, a lipid-soluble antioxidant, protected the mitochondrial function from honokiolinduced oxidative stress. Since many of the enzymes producing ROS are embedded in lipid bilayers and the unsaturated bond in polyunsaturated fatty acids is relatively weak compared to other saturated bonds, lipids often become prime targets of oxygen radicals [31]. In our previous study, honokiol exposure induced a significant increase in the level of lipid peroxidation [10]. A supplement with VC exacerbated mitochondrial lipid peroxidation (Figs. S2 and 2). Lipid peroxides alter vital mitochondrial functions, such as respiration, oxidative phosphorylation, and ion transport [1]. Why is the antioxidant activity of VC transformed into pro-oxidant activity in the presence of honokiol? Honokiol, having two phenolic hydroxyl groups, could chelate ferric iron ion and cause intracellular iron starvation [32]. Honokiol-induced growth deficiency could be rescued by ferric iron ion supple- mentation but not by the addition of ferrous iron ion [32]. Due to the poor production of intracellular ferric iron ion after honokiol exposure, it can be hypothesized that excessive free iron would release from the iron pool and iron-containing proteins such as ferredoxin and Fe-Scontaining proteins. Hence, the antioxidant activity of VC could turn out to be pro-oxidant activity in the presence of ferric iron ion due ti the production of hydroxyl and alkoxyl radicals via Fenton reaction [33, 34]. In addition, several earlier articles had hinted that VC could interact with iron ions in the culture media leading to the production of $\mathrm{H}_{2} \mathrm{O}_{2}[35,36]$. VE, an efficient chain-breaking antioxidant in biological systems, is specifically incorporated into biological membranes, especially the inner mitochondrial membrane which hosts the enzymes of the respiratory electron transport chain [37]. Our previous data indicated that honokiol may target mitochondrial respiratory chain complex I located in the mitochondrial inner membrane, leading to mitochondrial dysfunction [10]. In our present study, VE supplement could effectively prevent honokiol-induced lipid peroxidation of the 
mitochondrial membrane, indicating the better ability of lipid-soluble VE to prevent lipid peroxidation of the mitochondrial membrane compared to that of watersoluble VC (Figs. S2 and 2).

It is reported that VC not only induces high ROS production and oxidative stress but also interferes with glycolytic metabolism by inhibiting GAPDH glycolytic enzyme activity $[6,38]$. Our previous data demonstrated that honokiol treatment suppresses mitochondrial respiration and oxidative phosphorylation, and increases ROS generation in the mitochondria, leading to the induction of apoptosis in C. albicans cells [24]. Due to the impaired mitochondrial function induced by honokiol exposure, C. albicans cells may be highly sensitive to glycolytic inhibitors such as VC and 2-DG (Figs. 4 and 5). Therefore, VC-supplemented honokiol will more severely target cell metabolism and energy production, thereby inhibiting the growth of $C$. albicans.

In summary, our study showed that VC potentiated the antifungal activities of honokiol whereas VE hindered the efficacy of honokiol treatment by exerting protective effects on mitochondria. Therefore, VC-supplemented honokiol could be used effectively to fight against $C$. albicans.

\section{Acknowledgments}

This work was supported by grants from the National Natural Science Foundation of China (81302814 and 81703574), the Jiangsu Province Natural Science Foundation (BK20130640 and BK20140624), and the Fundamental Research Funds for the Central Universities (No.2242019K40132).

\section{Conflict of Interest}

The authors have no financial conflicts of interest to declare.

\section{Reference}

1. Ott M, Gogvadze V, Orrenius S, Zhivotovsky B. 2007. Mitochondria, oxidative stress and cell death. Apoptosis 12: 913-922.

2. Villanueva C, Kross RD. 2012. Antioxidant-induced stress. Int. J. Mol. Sci. 13: 2091-2109.

3. Ayer A, Gourlay CW, Dawes IW. 2014. Cellular redox homeostasis, reactive oxygen species and replicative ageing in Saccharomyces cerevisiae. FEMS Yeast Res. 14: 60-72.

4. Traber MG, Stevens JF. 2011. Vitamins C and E: beneficial effects from a mechanistic perspective. Free Radic. Biol. Med. 51: 1000-1013.
5. Buettner GR. 1986. Ascorbate autoxidation in the presence of iron and copper chelates. Free Radic. Res. Commun. 1: 349-353.

6. De Francesco EM, Bonuccelli G, Maggiolini M, Sotgia F, Lisanti MP. 2017. Vitamin C and doxycycline: a synthetic lethal combination therapy targeting metabolic flexibility in cancer stem cells (CSCs). Oncotarget 8: 67269-67286.

7. Amorati R, Zotova J, Baschieri A, Valgimigli L. 2015. Antioxidant activity of magnolol and honokiol: kinetic and mechanistic investigations of their reaction with peroxyl radicals. J. Org. Chem. 80: 10651-10659.

8. Liao K, Sun L. 2018. The Roles of Hsp90-calcineurin pathway in the antifungal activity of honokiol. J. Microbiol. Biotechnol. 28: 1086-1093.

9. Sun LM, Liao K. 2018. Saccharomyces cerevisiae Hog1 MAP kinase pathway is activated in response to honokiol exposure. J. Appl. Microbiol. 124: 754-763.

10. Sun L, Liao K, Hang C, Wang D. 2017. Honokiol induces reactive oxygen species-mediated apoptosis in Candida albicans through mitochondrial dysfunction. PLoS One 12: e172228.

11. Sun L, Liao K, Wang D. 2017. Honokiol induces superoxide production by targeting mitochondrial respiratory chain complex I in Candida albicans. PLoS One 12: e184003.

12. Sun L, Liao K, Wang D. 2015. Effects of magnolol and honokiol on adhesion, yeast-hyphal transition, and formation of biofilm by Candida albicans. PLoS One 10: e117695.

13. Clinical and Laboratory Standards Institute (CLSI). 2008. M27-A3, Reference Method for Broth Dilution Antifungal Susceptibility Testing of Yeasts: Approved Standard, 3rd ed. Wayne, PA: Clinical and Laboratory Standards Institute.

14. Klepser ME, Wolfe EJ, Jones RN, Nightingale $\mathrm{CH}$, Pfaller MA. 1997. Antifungal pharmacodynamic characteristics of fluconazole and amphotericin B tested against Candida albicans. Antimicrob. Agents Chemother. 41: 1392-1395.

15. Takahashi M, Shibata M, Niki E. 2001. Estimation of lipid peroxidation of live cells using a fluorescent probe, diphenyl1-pyrenylphosphine. Free Radic. Biol. Med. 31: 164-174.

16. Cossarizza A, Salvioli S. 2001. Flow cytometric analysis of mitochondrial membrane potential using JC-1. Curr. Protoc. Cytom. Chapter 9: unit 9-14.

17. Smiley ST, Reers M, Mottola-Hartshorn C, Lin M, Chen A, Smith TW, et al. 1991. Intracellular heterogeneity in mitochondrial membrane potentials revealed by a Jaggregate-forming lipophilic cation JC-1. Proc. Natl. Acad. Sci. USA 88: 3671-3675.

18. Sun L, Zhao Y, Yuan H, Li X, Cheng A, Lou H. 2011. Solamargine, a steroidal alkaloid glycoside, induces oncosis in human K562 leukemia and squamous cell carcinoma KB cells. Cancer Chemother. Pharmacol. 67: 813-821.

19. Chang W, Zhang M, Li Y, Lou H. 2015. Flow cytometrybased method to detect persisters in Candida albicans. Antimicrob. Agents Chemother. 59: 5044-5048.

20. Morita M, Naito Y, Yoshikawa T, Niki E. 2016. Plasma lipid oxidation induced by peroxynitrite, hypochlorite, lipoxygenase 
and peroxyl radicals and its inhibition by antioxidants as assessed by diphenyl-1-pyrenylphosphine. Redox Biol. 8: 127-135.

21. Dellinger M, Geze M. 2001. Detection of mitochondrial DNA in living animal cells with fluorescence microscopy. J. Microsc. 204: 196-202.

22. Yun J, Mullarky E, Lu C, Bosch KN, Kavalier A, Rivera K, et al. 2015. Vitamin C selectively kills KRAS and BRAF mutant colorectal cancer cells by targeting GAPDH. Science 350: 1391-1396.

23. Pelicano H, Martin DS, Xu RH, Huang P. 2006. Glycolysis inhibition for anticancer treatment. Oncogene 25: 4633-4646.

24. Wisplinghoff H, Seifert H, Wenzel RP, Edmond MB. 2003. Current trends in the epidemiology of nosocomial bloodstream infections in patients with hematological malignancies and solid neoplasms in hospitals in the United States. Clin. Infect. Dis. 36: 1103-1110.

25. Bondaryk M, Kurzatkowski W, Staniszewska M. 2013. Antifungal agents commonly used in the superficial and mucosal candidiasis treatment: mode of action and resistance development. Postepy Dermatol. Alergol. 30: 293-301.

26. Teodoro GR, Ellepola K, Seneviratne CJ, Koga-Ito CY. 2015. Potential use of phenolic acids as anti-Candida agents: a review. Front Microbiol. 6: 1420.

27. Sun L, Hang C, Liao K. 2018. Synergistic effect of caffeic acid phenethyl ester with caspofungin against Candida albicans is mediated by disrupting iron homeostasis. Food Chem. Toxicol. 116: 51-58.

28. Sun L, Liao K, Hang C. 2018. Caffeic acid phenethyl ester synergistically enhances the antifungal activity of fluconazole against resistant Candida albicans. Phytomedicine 40: 55-58.
29. Labriola D, Livingston R. 1999. Possible interactions between dietary antioxidants and chemotherapy. Oncology (Williston Park) 13: 1003-1008,

30. Belhachemi MH, Boucherit K, Boucherit-Otmani Z, Belmir S, Benbekhti Z. 2014. Effects of ascorbic acid and alphatocopherol on the therapeutic index of amphotericin B. J. Mycol. Med. 24: e137-e142.

31. Yin $\mathrm{H}, \mathrm{Xu}$ L, Porter NA. 2011. Free radical lipid peroxidation: mechanisms and analysis. Chem. Rev. 111: 5944-5972.

32. Zhu X, Zou S, Li Y, Liang Y. 2017. Transcriptomic analysis of Saccharomyces cerevisiae upon honokiol treatment. Res. Microbiol. 168: 626-635.

33. Stohs SJ, Bagchi D. 1995. Oxidative mechanisms in the toxicity of metal ions. Free Radic. Biol. Med. 18: 321-336.

34. Carr A, Frei B. 1999. Does vitamin C act as a pro-oxidant under physiological conditions? FASEB J. 13: 1007-1024.

35. Clement MV, Ramalingam J, Long LH, Halliwell B. 2001. The in vitro cytotoxicity of ascorbate depends on the culture medium used to perform the assay and involves hydrogen peroxide. Antioxid. Redox Signal. 3: 157-163.

36. Halliwell B. 2013. The antioxidant paradox: less paradoxical now? Br. J. Clin. Pharmacol. 75: 637-644.

37. Gohil K, Packer L, de Lumen B, Brooks GA, Terblanche SE. 1986. Vitamin E deficiency and vitamin C supplements: exercise and mitochondrial oxidation. J. Appl. Physiol. 60: 1986-1991.

38. Bonuccelli G, De Francesco EM, de Boer R, Tanowitz HB, Lisanti MP. 2017. NADH autofluorescence, a new metabolic biomarker for cancer stem cells: Identification of vitamin C and CAPE as natural products targeting "stemness". Oncotarget 8: 20667-20678. 